\title{
Gaia 8: Discovery of a star cluster containing $\beta$ Lyrae
}

\author{
U. Bastian \\ Zentrum für Astronomie (Center for Astronomy), Heidelberg University, Mönchhofstr. 14, 69120 Heidelberg, Germany
e-mail: bastian@ari . uni-heidelberg. de
}

Received 28 August 2019 / Accepted 11 September 2019

\begin{abstract}
Aims. The aim here is to try and clarify whether there are stars in the neighbourhood of $\beta$ Lyrae which are physically connected to the famous prototype eclipsing binary.

Methods. We scrutinised the astrometric and photometric data (from HIPPARCos and Gaia DR2) of stars in a limited sky field around $\beta$ Lyrae.

Results. A star cluster of about 100 members was discovered, nicely centred on $\beta$ Lyrae in five dimensions. There are clear indications that the newly discovered cluster belongs to a larger old (extinct) star formation complex, including two known star clusters and an extended, strewn field of stars at practically the same distance and space velocity.

Conclusions. This discovery opens up the possibility of determining a precise distance and precise age for $\beta$ Lyrae using the combined properties of the cluster members. This in turn may form an important basis for an improved astrophysical interpretation of the model binary $\beta$ Lyrae and its kin. The larger old star formation complex is of scientific interest in its own right.
\end{abstract}

Key words. binaries: general - stars: individual: $\beta$ Lyrae - open clusters and associations: individual: Gaia 8 open clusters and associations: individual: Stephenson 1 - open clusters and associations: individual: KPR2005 100

\section{Introduction}

The bright eclipsing binary $\beta$ Lyrae is an object of considerable historical and astrophysical interest. The SIMBAD database bears witness to the star's scientific importance, listing almost a thousand $^{1}$ literature references. The star is the prototype of evolved semi-detached binaries with strong mutual gravitational deformation of the partners, with mass transfer, significant period evolution, and occasionally an accretion disk around the receiving partner.

There is a long-running, low-level discussion debating whether or not some members of a group of a half-dozen stars of apparent magnitudes 7-11 around $\beta$ Lyrae might be physically connected to the massive binary; see for example Abt et al. (1962) and Abt \& Levy (1976). With the help of the astrometry and photometry of Gaia DR2, the second data release of the astrometry mission of ESA (Gaia Collaboration 2018; Lindegren et al. 2018), a final answer to this question could be expected. Checking the relevant data in DR2 surprisingly revealed an open star cluster of around 100 members to which $\beta$ Lyrae obviously belongs.

It is suggested to denote this cluster as Gaia 8, in reminiscence of the discovery of the clusters Gaia 1 (the surprising massive cluster next to Sirius on the sky) and Gaia 2 (in Perseus at more than $5 \mathrm{kpc}$ distance) by Koposov et al. (2017), and in honor of the Gaia astrometry satellite project which made these discoveries possible. The number 8 is given to the new cluster because meanwhile Torrealba et al. (2019) discovered and named clusters Gaia 3, 4, 5, 6, and 7 (still using Gaia DR1).

Sections 2 and 3 of the present Letter describe the cluster and its discovery, Sect. 4 presents its probable connection with an old

\footnotetext{
1 Precisely 878 as of August 11, 2019.
}

(extinct), extended, and hitherto unknown star formation complex, and Sect. 5 briefly discusses the possible scientific significance of the membership of $\beta$ Lyrae to the cluster, as well as the possible scientific interest of the surrounding larger stellar aggregate.

\section{Discovery of the cluster}

In order to check for objects possibly connected to $\beta$ Lyrae, initially a sky field of $1^{\circ}$ radius was retrieved from the public DR2 installation at Heidelberg ${ }^{2}$, the star list being restricted to significant DR2 parallaxes $\left(\varpi / \sigma_{\varpi}>8\right)$ and to brightness $G<20$. The proper-motion vector diagram of this sample is shown as red dots in Fig. 1. Further down-selecting on proper motions within \pm 1 mas/a around the HIPPARCos (van Leeuwen 2007) motion of $\beta$ Lyrae (blue box in Fig. 1) and parallaxes within \pm 1 mas around the HIPPARCos value for $\beta$ Lyrae led to the sample shown as green dots in Fig. 1. Surprisingly, that sample turned out to be clearly concentrated on the sky at the position of $\beta$ Lyrae (not shown, but see Fig. 4), and even more so in the upper-right corner of the original proper-motion box in Fig. 1. The immediately obvious suggestion of a star cluster was verified by dropping the parallax criterion and selecting a re-centred proper-motion sample (pink in Fig. 1). This resulted in a highly telling colourmagnitude diagram (Fig. 2), showing a very well-defined main sequence of nearby stars of equal distances (around $290 \mathrm{pc}$ ), plus a well-separated, unrelated galactic background. Due to the small sky field, there are no foreground stars in the selected proper-motion range.

HIPPARCos rather than DR2 parameters for $\beta$ Lyrae were used here for the following reasons: Gaia can only measure this

\footnotetext{
2 See http://gaia.ari.uni-heidelberg.de/tap.html
} 


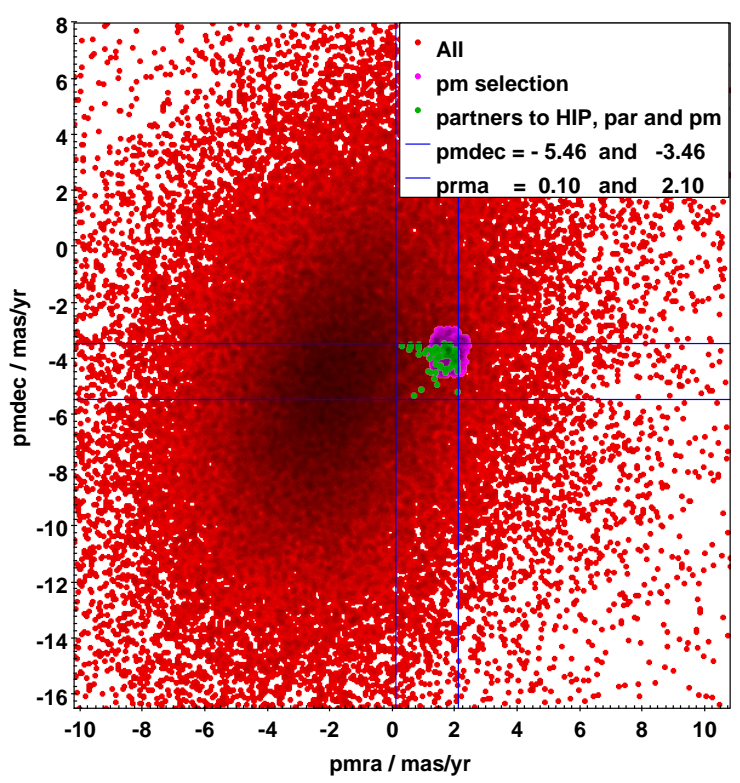

Fig. 1. Proper-motion vector diagram of the Gaia DR2 stars in a circle of $1^{\circ}$ radius around $\beta$ Lyrae. The blue lines and the two sub-samples which led to the discovery of the star cluster are explained in the text. Axes are labelled by the names of the relevant quantities in the public Gaia DR2 tables; units are indicated.

extremely bright star ( $G$ magnitude about 3.2-4.4) in the form of strongly saturated images (see Gaia Collaboration 2016, and references therein). For Gaia DR2 (Gaia Collaboration 2018; Lindegren et al. 2018) it has thus far been impossible to sufficiently calibrate ${ }^{3}$ these images astrometrically. This can be seen from the overall error distribution of stars with $G<5$ in DR2 as well as from some strange individual results for such very bright stars. In addition, a number of very bright stars are missing in the catalogue.

The particular star $\beta$ Lyrae itself is present in DR2, but its $G$ magnitude in DR2 is given as 7.5, its parallax of 1.1 mas is clearly in discordance with the HIPPARCos parallax of 3.4 mas, and its astrometric quality indicators can best be summarised as "terrible" 4

\section{Confirmation of the cluster and of the membership of $\beta$ Lyrae}

A confirmation of the cluster which is completely independent of the proper motions and photometric data used for its discovery can be provided by the distribution of the Gaia DR2 parallaxes. To this purpose, Fig. 3 uses the same star sample as Fig. 2. The cluster is robustly confirmed; the mean parallax of the obvious candidate members is about 3.4 mas. The scatter of the parallaxes at the bright end of Fig. 3 is clearly larger than the indicated DR2 parallax uncertainties. This is not due to measurement errors, but nicely agrees with the putative spatial depth of the cluster: The angular radius of slightly under $1^{\circ}$ (see Fig. 4) corresponds to $5 \mathrm{pc}$ at a parallax of $\varpi=3.4$ mas, which trans-

\footnotetext{
3 We note that Gaia was designed and announced for a bright magnitude limit of $G=5.7$, i.e. $\beta$ Lyrae is $3-10$ times brighter than this limit.

4 Specifically (with typical values for stars of magnitude $G<$ 9 given in parentheses): astrometric_gof_al=237 (ca. 5); astrometric_excess_noise $=2.1$ mas (mostly zero); parallax_error $=0.34$ mas (0.04 mas); phot_bp_rb_excess_factor $=4.8$ (1.2).
}

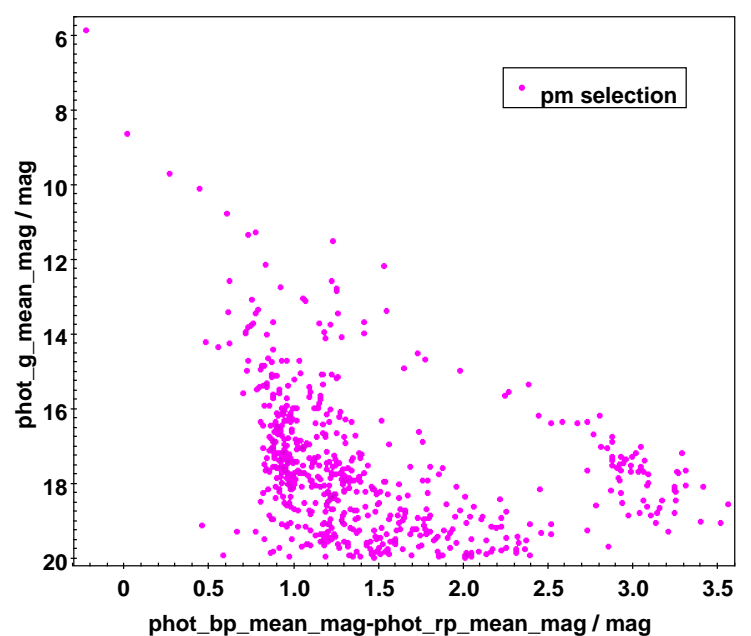

Fig. 2. Straight colour-magnitude diagram of the stars in the pink area of the proper-motion vector diagram of Fig. 1. The star cluster is indicated by the very clear-cut main sequence of close-by stars, well separated from a set of unrelated galactic background stars incidentally having the same proper motion. Axes are labelled by the names of the relevant quantities in the public Gaia DR2 tables; units are indicated.

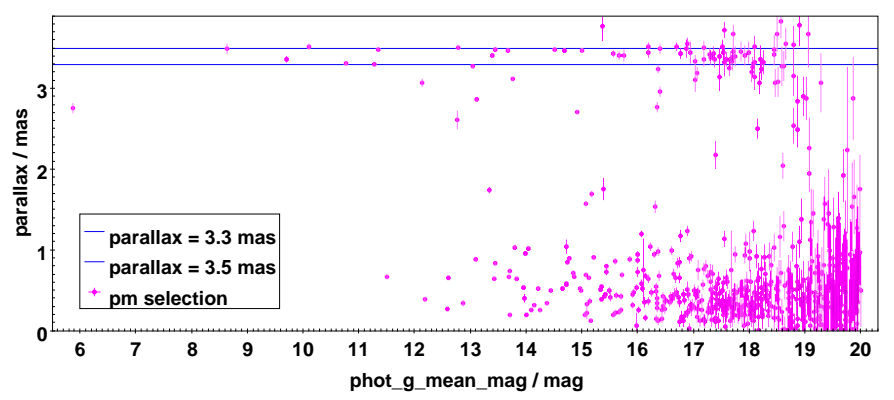

Fig. 3. Gaia DR2 parallaxes of the star sample in Fig. 2, independently confirming the cluster nature of the photometric discovery made in view of Fig. 2. The horizontal axis is the Gaia DR2 $G$ magnitude; axes are labelled with the names of the relevant quantities in the public Gaia DR2 tables; units are indicated. The pink bars indicate the standard errors of the parallaxes, as given in DR2.

lates into $\Delta \varpi=0.06$ mas in radial direction. Using the individual DR2 parallaxes to derive absolute magnitudes and plotting an "HRD", an extremely sharp main sequence is created (see Fig. 5).

The cluster membership of $\beta$ Lyrae is made virtually certain by the following facts:

- The cluster is perfectly centred on $\beta$ Lyrae on the sky. This is to be expected if $\beta$ Lyrae is by far the heaviest member.

- The proper motion of the cluster is fully consistent with the HIPPARCOS motion of $\beta$ Lyrae.

- The mean parallax of the candidate members is practically equal to the HIPPARCos value for $\beta$ Lyrae.

- There are four candidate members with radial velocities given in Gaia DR2: three of them cluster around $-17 \mathrm{~km} \mathrm{~s}^{-1}$, the fourth one is at $+5 \mathrm{~km} \mathrm{~s}^{-1}$. The General Catalogue of Stellar Radial Velocities (1953) lists $\beta$ Lyrae at $-19 \mathrm{~km} \mathrm{~s}^{-1}$, while Abt et al. (1962) independently found $-18.5( \pm 1) \mathrm{km} \mathrm{s}^{-1}$.

\section{Investigation of the neighbourhood of the cluster}

Closer inspection of Fig. 3 reveals that - compared to a pure field-star distribution - there is a clear excess of stars at slightly 


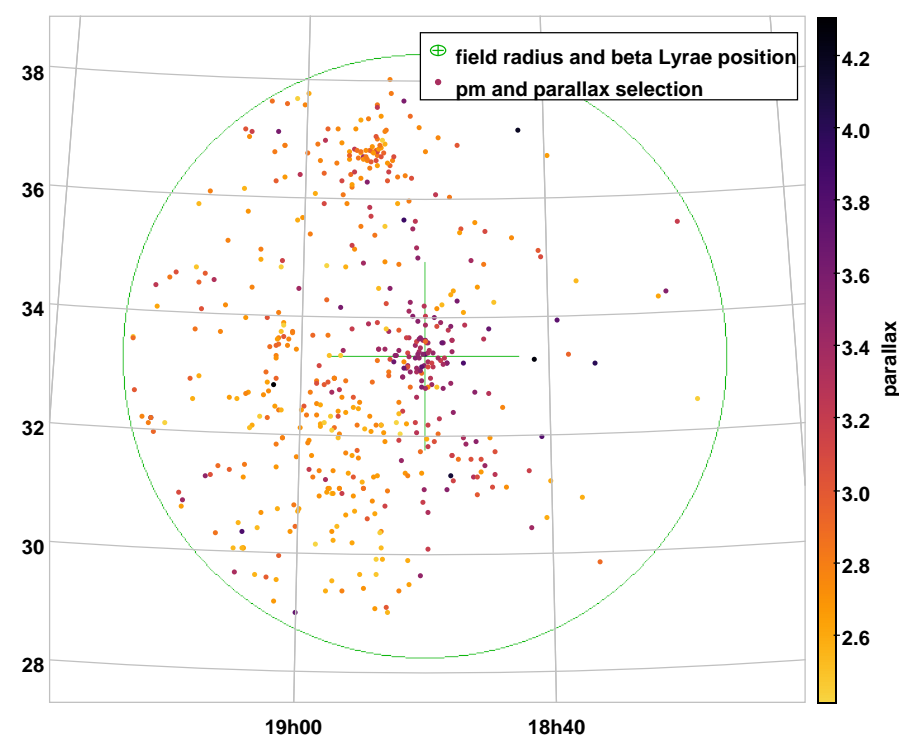

Fig. 4. Sky distribution of the stars within 1 mas/a in proper motion and 1 mas in parallax around the motion and parallax of the cluster containing $\beta$ Lyrae, and within $5^{\circ}$ angular separation around the position of $\beta$ Lyrae itself (green cross). The individual star symbols are coloured by the DR2 parallaxes. Four stellar aggregates can be seen, as described in the text.

smaller parallax than the $\beta$ Lyrae cluster. Is there another cluster? To investigate this question, an analogous star sample to that of Fig. 2 was created, but on a larger sky field with $5^{\circ}$ in radius, and adding the \pm 1 mas parallax criterion around the 3.4 mas value of the $\beta$ Lyrae cluster. A simple sky map of this sample is given in Fig. 4. Four features are evident:

- The prominent cluster Gaia 8, perfectly centred on the position of $\beta$ Lyrae, with parallaxes around 3.4 mas (violetbrownish colours of the plotted dots).

- Another prominent cluster near the top of the map, with parallaxes around 2.7 mas (orange-yellowish colours). This is Stephenson 1 alias $\delta$ Lyrae cluster.

- A less prominent cluster about $2^{\circ}$ east of $\beta$ Lyrae, also with parallaxes around 2.7 mas (orange-yellowish colours). This is KPR2005 100 alias ASCC 100; see Kharchenko et al. (2005) and Kharchenko et al. (2007).

- An extended strewn field of stars southeast of $\beta$ Lyrae and possibly overlapping with Gaia 8 , again with parallaxes around 2.7 mas and slightly smaller. This is a real stellar agglomeration: From an equivalent skymap avoiding the parallax selection (not shown) it can be definitively excluded that the enhanced density of selected stars in the bottom-left part of Fig. 4 is due to interstellar extinction at the right-hand half of the map, or due to the lower galactic latitude at the bottom left of the map.

The immediate suggestion that these four features might be genealogically connected, that is that they might constitute parts of a larger old (extinct) star formation complex, was investigated by analysing manual ad-hoc selections of the candidate members of each feature around their respective centres in Fig. 4. An intimate connection among them is revealed to be almost certain by the following facts:

- All four are located within a very small region of the galactic disk: The largest tangential distance is between the centres of Stephenson 1 and of the strewn field. This is about $30 \mathrm{pc}$ at an assumed parallax of 2.75 mas. The maximum radial distance is given by the difference in mean parallax between Gaia 8

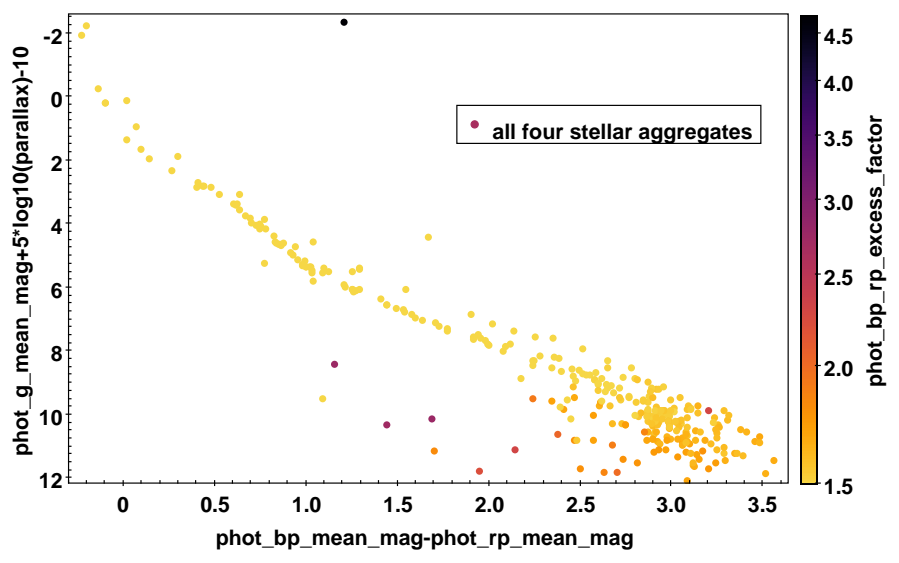

Fig. 5. Combined "HRD" for the manually selected candidate members of the four stellar groups identified in Fig. 4. The symbols are coloured according to the so-called BP-RP flux excess from Gaia DR2. For a precise definition and for extensive explanations, see the DR2 documentation. In short: High values of this quantity indicate unresolved double stars with disturbed Gaia DR2 colours. This quantity is shown here in order to indicate that the single dark dot at the top is not due to a real giant belonging to the cluster(s), but to a very probably incorrect colour. Similarly, most of the candidate members far left of the main sequence at the bottom are very probably true members, but with disturbed colours.

and the other three, that is about $70 \mathrm{pc}$. Those other three differ by less than $20 \mathrm{pc}$ in distance, which is less than the on-sky diameter of the strewn field.

- All four share almost the same tangential velocity relative to the sun. Although their respective mean tangential velocities differ significantly statistically (due to the high precision of Gaia DR2), they are all less than $1 \mathrm{~km} \mathrm{~s}^{-1}$ from that of Gaia 8. As side note, the proper motion of Gaia 8 nevertheless differs more strongly from the other three, due to the smaller distance.

- All four share the same radial velocity. Among the 521 stars in Fig. 4 there are 43 with a radial velocity given in Gaia DR2. Among these, 41 are consistent with a uniform velocity of $-18 \mathrm{~km} \mathrm{~s}^{-1}$. They are distributed over all four candidate member selections.

- A combined "HRD" (Fig. 5) for all four selections (using the individual DR2 parallaxes per star to derive absolute $G$ magnitudes) shows an extremely uniform and sharp main sequence, along with a well-defined binary sequence running in parallel. The narrowness of this main sequence points to a similar age and metallicity of the entire complex. It is a pity that no genuine subgiants or giants belong to the complex; otherwise, a precise age could directly be derived from Fig. 5. An age between 30 and $100 \mathrm{Myr}$ is indicated, but this remains to be confirmed by a quantitative isochrone fitting (in a follow-up study).

Another informative view of the contents of Fig. 4 is given by Fig. 6, which is an equivalent of Fig. 3, but now for the same star selection as in Fig. 5, and with the individual symbols coloured according to the manual membership selection of the four stellar groups discussed above. It can be seen that the originally "disturbing" parallaxes from Fig. 3 are actually members of the strewn field superposed on the cluster Gaia 8 (pink dots in the lower band of symbols), while a few stars assigned to other aggregates (from the purely position-based sample selections) are in fact outlying members of Gaia 8 (blue and green dots in the upper band of symbols). The latter are also directly apparent 


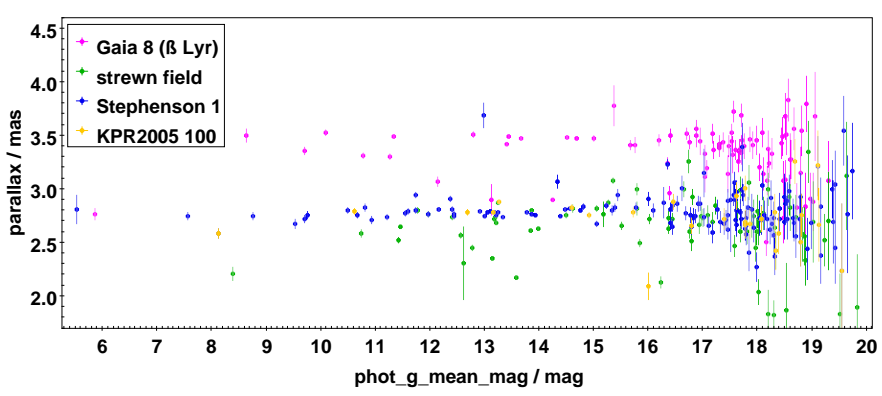

Fig. 6. Gaia DR2 parallaxes of candidate members of the three clusters and the strewn field of Fig. 4. The horizontal axis is the Gaia DR2 $\mathrm{G}$ magnitude; axes are labelled by the names of the relevant quantities in the public Gaia DR2 tables; units are indicated. The bars indicate the standard errors of the parallaxes, as given in DR2. Colours denote tentative membership assignments; see text.

in Fig. 4 as loosely scattered dark dots. In addition, Fig. 6 clearly shows the difference in distance between Gaia 8 and the other three stellar groups, as well as the similarity of the distance among these three - plus the presence of a few stars in the strewn field with larger distances (green dots at the bottom). Some of them may be unrelated background stars, particularly the single yellow dot at parallax $\simeq 2.1$ mas and $G \simeq 16$.

\section{Discussion}

Clearly, the newly found cluster Gaia 8 as well as the larger stellar aggregate merit further investigation. The astrometric part of Gaia DR3 - providing more-precise parallaxes by about a factor of 1.25 and more-precise motions by almost a factor of $2-$ is due to be published in less than a year. Therefore it seems reasonable to use that release for this purpose. The main aim of the present short paper is to prompt the collection of supportive detailed observational data on some members in the meantime. Lists of candidate members will be provided by the author on request.

The scientific motivation for more detailed investigations will stem - on the one hand - from the astrophysical interest in $\beta$ Lyrae itself. Using precise multi-colour photometry in combination with high-quality spectroscopy of a small number of bright single-star members, or for example asteroseismology of just one or two bright members, can provide reliable estimates of the age and metallicity of the cluster. This in turn will form a very useful basis for an improved astrophysical interpretation of the model binary $\beta$ Lyrae and its kin.
On the other hand, the larger stellar aggregate can serve as an interesting case study for the long-term future of extended, diffuse star formation regions, including double clusters and so on. Several such similar groups and complexes of stars and clusters have been identified since 2018 (all from Gaia data), by for example Cantat-Gaudin et al. (2019), Kounkel \& Covey (2019), and possibly by Franciosini et al. (2018). There is no doubt that significant progress will be made on this interesting topic in the near future.

Gaia 8, ASCC 100, Stephenson 1, and the sparser, more strewn field (i.e. all the stars displayed in Figs. 4-6) apparently form a subgroup of the coeval complex labelled "Stephenson 1" in Fig. 10 of Kounkel \& Covey (2019). Assuming that the entire complex shares a common age, they estimate $\log ($ age $) \simeq 7.4$ (25 Myr). This can surely be improved by using Gaia DR3 and the above-mentioned supportive observational data, especially for the particularly interesting cluster containing $\beta$ Lyrae itself.

Acknowledgements. This work is based on data from the European Space Agency (ESA) mission Gaia (https://www.cosmos.esa.int/gaia), processed by the Gaia Data Processing and Analysis Consortium (DPAC, https: //www.cosmos.esa.int/web/gaia/dpac/consortium). Funding for the DPAC has been provided by national institutions, in particular the institutions participating in the Gaia Multilateral Agreement. This research has made use of the SIMBAD database, operated by the CDS at Strasbourg, France. The plots in this paper were produced using the TOPCAT tool, created and provided by Mark Taylor (Bristol University). The author thanks Wolfgang Quester for pointing him to the old question on possible stellar partners of $\beta$ Lyrae. Furthermore, many thanks go to an anonymous referee. He/she made a few suggestions which definitely improved the paper.

\section{References}

Abt, H., \& Levy, S. 1976, AJ, 81, 659

Abt, H., Jeffers, H. M., Gibson, J., \& Sandage, A. R. 1962, ApJ, 135, 429

Cantat-Gaudin, T., Jordi, C., Wright, N. J., et al. 2019, A\&A, 626, A17

Franciosini, E., Sacco, G. G., Jeffries, R. D., et al. 2018, A\&A, 616, L12

Gaia Collaboration (Prusti, T., et al.) 2016, A\&A, 595, A1

Gaia Collaboration (Brown, A. G. A., et al.) 2018, A\&A, 616, A1

Kharchenko, N. V., Piskunov, A. E., Roeser, S., Schilbach, E., \& Scholz, R.-D. 2005, A\&A, 440, 403

Kharchenko, N. V., Scholz, R.-D., Piskunov, A. E., Röser, S., \& Schilbach, E. 2007, Astron. Nachr., 328, 889

Koposov, D., Belokurov, V., \& Torrealba, G. 2017, MNRAS, 470, 2702

Kounkel, M., \& Covey, K. 2019, AJ, 158, 122

Lindegren, L., Hernández, J., Bombrun, A., et al. 2018, A\&A, 616, A2

Torrealba, G., Belokurov, V., \& Koposov, D. 2019, MNRAS, 484, 2181

van Leeuwen, F. 2007, in Hipparcos, the New Reduction of the Raw Data, Astrophys. Space Sci. Lib., 350 